OPEN ACCESS

Edited by:

Vassiliki A Boussiotis, Beth Israel Deaconess Medical Center and Harvard Medical School,

United States

Reviewed by:

Gregory B. Lesinski,

Emory University, United States

Amorette Barber,

Longwood University, United States

${ }^{*}$ Correspondence:

Robert H. Vonderheide rhv@upenn.edu

Specialty section: This article was submitted to Cancer Immunity and Immunotherapy,

a section of the journal

Frontiers in Immunology

Received: 12 September 2020

Accepted: 15 October 2020

Published: 10 November 2020

Citation:

Vonderheide RH and Bear AS (2020)

Tumor-Derived Myeloid Cell

Chemoattractants and T Cell Exclusion

in Pancreatic Cancer.

Front. Immunol. 11:605619.

doi: 10.3389/fimmu.2020.605619

\section{Tumor-Derived Myeloid Cell Chemoattractants and T Cell Exclusion in Pancreatic Cancer}

\author{
Robert H. Vonderheide ${ }^{*}$ and Adham S. Bear \\ Abramson Cancer Center, Department of Medicine, Perelman School of Medicine, University of Pennsylvania, Philadelphia, \\ $P A$, United States
}

Like many tumor types, pancreatic ductal adenocarcinoma (PDAC) exhibits a rich network of tumor-derived cytokines and chemokines that drive recruitment of myeloid cells to the tumor microenvironment (TME). These cells, which include tumor-associated macrophages and myeloid derived suppressor cells, block the recruitment and priming of T cells, resulting in T cell exclusion within the TME. Genetic or pharmacologic disruption of this chemokine/cytokine network reliably converts the PDAC TME to a T cell-high phenotype and sensitizes tumors to immunotherapy across multiple preclinical models. Thus, neutralization of tumor-derived chemokines/cytokines or blockade of their respective receptors represents a potentially potent strategy to reverse myeloid immunosuppression in PDAC, enabling benefit from checkpoint inhibition not otherwise achievable in this disease. Inhibition of oncogenic pathways that drive tumor-intrinsic expression of chemoattractants may be similarly effective.

Keywords: myeloid, pancreatic, chemoattractant molecules, $\mathrm{T}$ cells, macrophages

\section{INTRODUCTION}

A cancer inflammatory reaction dominated by myeloid cells is characteristic of many tumors, especially oncogene-driven invasive adenocarcinomas such as pancreatic ductal adenocarcinoma (PDAC) (1). The immunosuppressive cellular network established by tumor-derived myeloid cell chemoattractants - and the prospect of targeting this network therapeutically - has been increasingly understood, although the initial landmark observations underlying this concept were made by Mantovani and colleagues more than 35 years ago (2).

Experimental scrutiny of genetic mouse models of PDAC has uncovered a network of tumorderived chemoattractants that promote myeloid cell infiltration from the earliest stages of tumorigenesis, and these observations have been validated to an increasing extent in PDAC patients. Myeloid cells in the tumor microenvironment (TME) block endogenous anti-tumor T cell responses and thwart effective utilization of checkpoint inhibitors. Given multiple redundancies in the myeloid compartment, in vivo depletion of myeloid cells in the TME in a clinically relevant fashion has been challenging. Disruption of this chemokine/cytokine network or the respective receptors may be more tractable with neutralizing antibodies or small molecular inhibitors that experimentally lead to loss of myeloid inflammation in the TME. Such strategies have resulted in effector $\mathrm{T}$ cell TME infiltration and $\mathrm{T}$ cell-dependent tumor regressions that can be further augmented with immunotherapy. Widespread clinical application of this strategy would require 
precision profiling of tumors to identify personalized targetable chemokine/cytokines or receptors. A potentially more generalizable and therapeutically effective alternative would be the development of inhibitors of the oncogenes that drive the tumor expression of these chemoattractants, e.g., inhibitors of mutant Kras. This review outlines the role of myeloid chemoattractants in promoting $\mathrm{T}$ cell exclusion in PDAC to establish immune privilege as well as suggests opportunities to improve cancer immunotherapy in scenarios where single-agent checkpoint blockade fails.

\section{PDAC RESISTANCE TO CHECKPOINT BLOCKADE}

Despite increasing label indications across numerous cancer histologies, antibodies that block CTLA-4, PD-1, and PD-L1 are essentially ineffective in patients with advanced metastatic PDAC, linked mechanistically to a number of tumor-intrinsic and -extrinsic factors in the TME (Table 1) (3). Combinations of anti-CTLA-4 and anti-PD-L1 have been equally disappointing (4). One exception are the $<1 \%$ of all PDAC patients with high microsatellite instability (5), who often can respond to PD-1 checkpoint blockade and for whom pembrolizumab is now FDAapproved. Clinical trials continue to test the combination of checkpoint blockade with chemotherapy in metastatic PDAC, but initial results of gemcitabine, nab-paclitaxel, and nivolumab are discouraging. Thus, PDAC represents one of the most refractory tumors to currently approved checkpoint therapies, a particularly disappointing situation given the dire unmet medical need for a cancer that kills more individuals now than breast cancer and is predicted to become the second-leading cause of cancer death by 2030 (6).

Numerous preclinical studies predicted the poor clinical activity of checkpoint inhibitors in PDAC patients. In the "KPC" genetically engineered mouse model of PDAC (in which mutant Kras and p53 are targeted for expression in the pancreas, resulting in a high fidelity model of the disease), there is no antitumor response in spontaneous tumors to single (CTLA-4, PD-1, PD-L1) or combination (CTLA-4 plus PD-1/PD-L1) immune checkpoint blockade $(7,8)$. In subcutaneous or orthotopic implantable models using KPC-derived, syngeneic tumor cell lines, responses to checkpoint blockade are only rarely observed (7). Classically, KPC tumors exhibit poor T cell infiltration and very low tumor mutational burden (TMB) that translates into few

TABLE 1 | Obstacles in pancreatic cancer limiting utility of checkpoint blockade.

\begin{tabular}{ll}
\hline Category & \multicolumn{1}{c}{ Factor } \\
\hline Tumor intrinsic & Relatively low tumor mutational burden and neo-epitopes \\
& Low tumor PD-L1 expression \\
& Low rate of MSI high tumors $(<1 \%)$ \\
T cell response & Relatively low T cell infiltration in most tumors \\
& Minimal baseline T cell priming against the tumor \\
Stroma & Dense stroma limiting drug delivery to TME \\
& Large inhibitory myeloid cell population \\
& Inhibitory cancer-associated fibroblasts
\end{tabular}

if any neo-epitopes (9-11). In other pancreatic models, where the TMB is higher, responses to checkpoint inhibition are observed at somewhat greater rates (12). These latter observations in nonKPC PDAC tumor models provide a rationale for the clinical evaluation of checkpoint blockade in PDAC patients. Despite the majority of human PDAC having low to very low $\mathrm{T}$ cell infiltration and TMB, about $20 \%$ of patients do exhibit $\mathrm{T}$ cell infiltration and a relatively elevated TMB, although there is no correlation between high $\mathrm{T}$ cell infiltration and high TMB (or neo-epitope burden) (13). The objective response rates in PDAC patients with single or dual checkpoint inhibition is far less than 20\%; thus, neither T cell infiltration nor TMB serve as adequate predictive biomarkers of response in PDAC. Furthermore, PD-L1 expression as determined by RNA sequencing of primary PDAC tumors is among the lowest for any of other well-described immune checkpoint molecules including CTLA-4, VISTA, TIM3, TIGIT, LAG3, ADORA2A, or IDO1 (13). Moreover, there is no difference in PD-L1 expression among $\mathrm{T}$ cellinfiltrated vs. non $\mathrm{T}$ cell-infiltrated human PDAC, in contrast to the other checkpoint molecules such as CTLA-4 for which expression is significantly higher in T cell-infiltrated tumors (13). These findings raise the hypothesis that PD-L1 does not serve as the critical immune checkpoint that drives immunosuppression in PDA, consistent with the observation that the addition of nivolumab to a promising cancer vaccine in advanced PDAC patients fails to improve overall survival (14).

\section{STRATEGIES TO SENSITIZE PDAC TO CHECKPOINT BLOCKADE}

Two primary strategies have been explored to sensitize PDAC patients to checkpoint inhibition (3). The first strategy hypothesizes that PDAC patients exhibit deficient $\mathrm{T}$ cell priming and a $\mathrm{T}$ cell response must first be mobilized to achieve efficacy with checkpoint inhibition (15). Immune priming strategies explored in combination with immune checkpoint inhibition include chemotherapy or radiation to induce immunogenic tumor cell death or the use of a cancer vaccine. This approach is highly effective in the KPC and other PDAC mouse models $(8,16,17)$. To date, in PDAC patients, the combination of chemotherapy or cancer vaccines with PD-1 has not shown synergy. In our experience using the KPC model, the addition of agonistic CD40 antibody, aimed at licensing dendritic cells to activate anti-tumor $\mathrm{T}$ cells, has been the single most potent method to sensitize tumor-bearing mice to PD-1, CTLA-4, or combination immune checkpoint blockade as has been recently reviewed (18). An ongoing national, randomized study is currently evaluating chemotherapy with or without agonistic $\mathrm{CD} 40 \mathrm{mAb}$, with or without nivolumab, in first-line metastatic PDAC patients, based on promising phase $1 \mathrm{~b}$ results with chemotherapy and $\mathrm{CD} 40 \mathrm{mAb}$ in the same patient population (NCT03214250). Other immune agonists such as those against stimulator of interferon genes (STING) or toll-like receptors (TLRs) represent additional approaches, as recently reviewed (3). 
The blockade of novel checkpoint molecules represent the second strategy to sensitize PDAC tumors to PD-1/PD-L1 inhibition. Many of these novel checkpoints are highly expressed in PDAC and as noted above, these molecules increase in expression in the TME of tumors with higher levels of infiltrating $\mathrm{T}$ cells (13). However, evidence for single-agent activity of antibodies targeting these novel checkpoints in PDAC to date been minimal or modest, although preclinical data with select inhibitors (e.g. VISTA) are compelling $(19,20)$. However, as a sobering reminder, no novel checkpoint inhibitors have been approved by the FDA in oncology since the initial approvals of anti-CTLA-4 and anti-PD-1 aside from variations of PD-1/PDL1 and combinations with anti-CTLA-4. As a telling example, the novel checkpoint inhibitor epacadostat (an IDO1 inhibitor) failed in combination with pembrolizumab in a randomized study in patients with advanced melanoma (21) despite compelling preclinical data in mice.

\section{THE MYELOID CHECKPOINT IN PDAC}

Leukocytes dominate the PDAC microenvironment and among these, myeloid cells are typically the most over-represented, contributing to the well-described picture of cancer inflammation and desmoplastic reaction in this disease (9). This phenotype is reproduced in the spontaneous KPC model, in which infiltration by macrophages and myeloid-derived suppressor cells (MDSC) is evident in neoplastic lesions even before tumor cell invasion (9). The immunosuppressive effect of MDSCs on T cells in particular is striking in the KPC model and can be demonstrated both ex vivo and in vivo $(22,23)$. In both the KPC model and human PDAC, there appears to be an inverse relationship between myeloid infiltration and effector $\mathrm{T}$ cell infiltration (13). Thus, the question remains - is there a causal relationship between myeloid infiltration and effector $\mathrm{T}$ cell paucity? In the KPC model, it has been difficult to discern pharmacologically if myeloid cells are obstructing $\mathrm{T}$ cell infiltration because methods to eliminate myeloid cells in vivo are at best incomplete and transient (8). An alternative approach is to activate myeloid cells and re-educate (rather than deplete) them away from tumor-promoting activities. Such activation can be accomplished with agonistic CD40 antibody in the KPC model (24). With chemotherapy or radiation therapy, CD40 antibody can produce $\mathrm{T}$-cell dependent tumor regressions (8, 16). Agonism of myeloid cell $\mathrm{CD} 11 \mathrm{~b}$ also repolarizes tumorassociated macrophages (TAM), reduces intratumoral myeloid cells, and leads to anti-tumor immunity in concert with checkpoint inhibition (25).

Myeloid cells in the TME are highly heterogeneous, and novel techniques such as ultra-high multiplex flow cytometry and single cell sequencing have unearthed significant complexities (26-28) which presents challenges for nomenclature systems to capture (29). Current views of myeloid heterogeneity extend far beyond designations of M1 vs M2 (or N1 vs N2); however, understanding the range of functionalities of various myeloid cells in the TME as either anti-tumor vs pro-tumor has been helpful conceptually. The rich mechanisms and dynamics that regulate myeloid cells and mechanisms of immunosuppression have been reviewed elsewhere $(28,30,31)$.

Further indication that myeloid cells represent a major immune checkpoint in PDAC comes from studies in which individual cancer cells from spontaneous KPC tumors were cloned, re-implanted in syngeneic hosts, and upon tumor outgrowth harvested and inspected for $\mathrm{T}$ cell and myeloid cell infiltration (32). Although T cell-high tumors are unusual among spontaneous KPC tumors, KPC clones upon re-implantation are frequently $\mathrm{T}$ cell-high in this experiment. Interestingly, $\mathrm{T}$ cellhigh tumors feature unusually poor myeloid infiltration, reproducing the human phenotype. In mixing studies administering $\mathrm{T}$ cell-low and $\mathrm{T}$ cell-high $\mathrm{KPC}$ clones, the $\mathrm{T}$ cell-low phenotype is dominant as outgrowing tumors are T celllow and myeloid-rich (32). T cell-high tumors themselves are strikingly sensitive to anti-PD-1 and anti-CTLA-4 checkpoint therapy, even in the absence of CD40 agonism or chemotherapy (32). On the contrary, T cell-low tumors are refractory to antiPD-1/anti-CTLA-4 recapitulating observations in the treatment of spontaneous KPC tumors.

\section{MYELOID CHEMOATTRACTANTS IN PDAC}

Observations of T cell-high and T cell-low KPC clonal mixing studies led to the hypothesis that a local chemo-attractant factor elaborated by $\mathrm{T}$ cell-low tumor clones drives recruitment of myeloid cells to the TME. These myeloid cells in turn inhibit T cells and block their recruitment (Figure 1). In addition to the inhibition of $\mathrm{T}$ cell recruitment and effector function, myeloid cells may also produce factors that hinder dendritic cells in the TME, thereby preventing effective priming of anti-tumor effector T cell responses. For example, IL- 6 has been shown to have such antagonistic effects on dendritic cells in PDAC (33). Consistent with this hypothesis, numerous studies have identified and characterized tumor-intrinsic pro-myeloid cytokines that regulate $\mathrm{T}$ cell immunosuppression in mouse models of PDAC. Examples include GM-CSF (22, 23), CXCR2 ligands (32, 34-36), CSF3 (32), CCR2 ligands (37), and CSF1 (38-40), as detailed below. Despite wide variation among cell lines as to which tumor-derived cytokine most prominently drives a myeloidrich TME, precise ablation of a single, dominant cytokine for any given cell line reliably leads to $\mathrm{T}$ cell influx and immunotherapy responsiveness. In each case, the tumor cells themselves (albeit, not necessarily only the tumor cells) elaborate the cytokine or chemokine which are downstream products of either oncogenic mutant Kras or other driver pathways. Thus, oncogenic pathways can also enforce a myeloid-rich TME in addition to promoting oncogenic survival, proliferation and invasion (41). Rapid myeloid domination of the TME occurs from the earliest stages of tumor inception (9). Lineage tracing in the KPC model which permits the identification of metastatic 

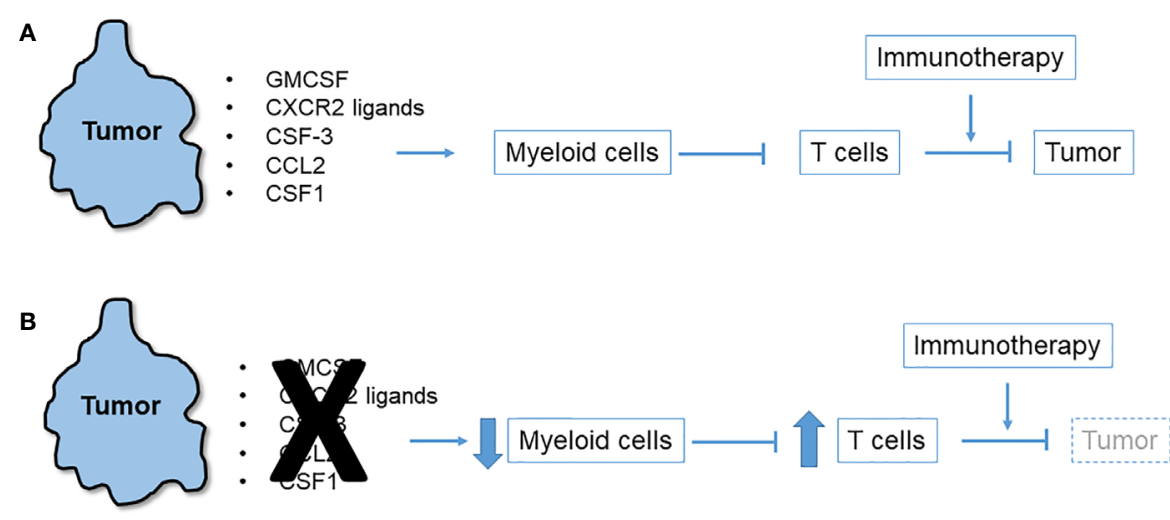

FIGURE 1 | Immunosuppressive network of tumor-derived myeloid cell chemoattractants. (A) Multiple chemokines and cytokines released by pancreatic tumor cells trigger influx of myeloid cells to the tumor microenvironment (TME) that in turn suppress T cells that could otherwise attack the tumor or be induced to do so with immunotherapy. (B) Blockade or neutralization of tumor-derived chemoattractants in numerous mouse models leads to diminution of myeloid cells in the TME, an upsurge in infiltrating $\mathrm{T}$ cells, and tumor regression especially after immunotherapy.

tumor cells as isolated singlets in liver and lung identifies macrophages as accompanying lone tumor cells (42).

\section{GM-CSF}

This growth factor is commonly expressed by tumor cells in both KPC mice and humans, even in early lesions (23). GM-CSF drives local proliferation of MDSC, which progressively accumulate in the PDAC microenvironment. When GM-CSF is genetically deleted from tumor cells in mice, $\mathrm{T}$ cell influx is triggered and tumors are rejected, but only in mice that are replete of CD8+ T cells $(22,23)$. GM-CSF is paradoxically understood to be a vaccine adjuvant and key component of promising pancreatic cancer vaccines (43), but as opposed to the low concentrations used in a subdermal vaccine, high concentrations of GM-CSF within the TME are immunosuppressive owing to effects on MDSC recruitment. In PDAC cells, GM-CSF production is downstream from mutant Kras signaling, linking immunosuppression to the driving oncogene (23).

\section{CXCR2 LIGANDS}

CXCR2 ligands regulate myeloid trafficking into tumor cells. In humans with PDAC, high expression of CXCR2 is correlated with enrichment of intra-tumoral neutrophils (34). In the KPC model, CXCR2 blockade by genetic or pharmacologic means reduces recruitment of myeloid cells into the PDAC microenvironment (especially neutrophils), permitting T-cell dependent suppression of tumor growth, an effect which can be augmented by PD-1 inhibition to improve survival $(34,35)$. Although CXCR2 is highly expressed by cells in the tumor stroma, tumor expression of CXCR2 has also been observed in various genetic models and may drive autocrine or paracrine growth $(44,45)$. In KPC experiments, CXCL5 is the most prominent CXCR2 ligand produced by tumor cells, whereas stromal cells produce CXCL2 (34). Expression of tumorderived CXCL5 is associated with mutant Kras expression and regulated by tumor NF-kB activation. In comparing $\mathrm{T}$ cell-high vs T cell-low KPC clones, CXCL1 - another CXCR2 ligand - is found to be the most differentially expressed (32). In these studies, CXCL2 and CXCL5, although known to be expressed in PDAC genetic models (45), are not differentially expressed. Genetic ablation of CXCL1 in T cell-low KPC clones abrogates the influx of myeloid cells in the TME, enabling infiltration by $\mathrm{CD} 8+\mathrm{T}$ cells and rendering tumors universally responsive to anti-CTLA-4, anti-PD-1, and CD40 combination therapy (32). In contrast, overexpression of CXCL1 in T cell-high tumors reverses this phenotype and blunts response to immunotherapy. Epigenetic variations acting in concert with MYC in T cell-low vs $\mathrm{T}$ cell-high tumors underlie the differential regulation of CXCL1 (32). CXCL1 expression in the KPC system also depends on the necrosome, and in a study of KPC orthotopic tumors, RIP-1/ RIP-3 driven necroptosis upregulates tumor-derived CXCL1 production and enhances peritumoral MDSC infiltration (36). RIP deletion reduces MDSC and triggers an influx of T cells and subsequent tumor regression. CXCL1 blockade similarly reduces MDSC in the tumors (36). RIP3 deletion is not, however, protective in B16 melanoma or subcutaneously implanted KPC tumors, indicating needed caution in explanatory models so as not to mistakenly imply that a single chemokine pathway is applicable across models or histologies.

\section{CSF3}

Several studies also identify tumor-derived CSF3 (also known as G-CSF) as a cytokine that recruits myeloid cells to the TME, and CSF3 is associated with $\mathrm{T}$ cell inhibition and desensitization of PDAC tumors to immunotherapy $(22,32,45)$. 


\section{CCL2}

Also known as MCP-1, CCL2 is a well-known tumor-derived macrophage chemoattractant in the TME $(46,47)$. Both human PDAC and KPC tumor cells express high levels of CCL2, although normal cells also express this chemokine $(22,37,48)$. The elaboration of CCL2 in the TME results in mobilization of CCR2+ monocytes that are immunosuppressive. Although CCR2 inhibitors show promise in depleting TAMS in vivo in patients $(37,49)$, a compensatory mechanism of CXCR2+ neutrophils frustrates anti-tumor efficacy. The combination of both CCR2 and CXCR2 inhibitors in KPC mice prevents this compensatory reaction and results in significantly smaller tumors and improved survival, an effect that can be further enhanced with chemotherapy (50). As with other chemoattractants, the mechanism appears to be tumorintrinsic. In KPC tumors, the epigenetic regulator HDAC5 inhibits Socs3, a negative regulator of CCL2, promoting CCL2 secretion and the recruitment of tumor-promoting macrophages to the TME (51).

\section{CSF1}

Also known as M-CSF, CSF1 is commonly highly expressed by mutant Kras engineered mouse tumors $(22,38)$ and human PDAC cells. Inhibition of CSF1 or CSF1-R using blocking antibodies or small molecule inhibitors leads to the selective depletion of TAMs in the TME in pancreatic mouse tumor models $(38,39)$ and promotes tumor regression in combination with anti-PD- 1 therapy with or without cancer vaccination $(38,40)$.

\section{OTHER MYELOID CELL REGULATORS IN THE PDAC TME}

Non-cytokine, tumor-intrinsic mechanisms of myeloid cell regulation are also active in PDAC mouse models. The tyrosine kinase EPHA2, for example, is markedly overexpressed in human and murine PDAC and functions to enforce a myeloid-rich, T cell-low phenotype in the TME (52). Knock-out of EPHA2 in tumor cells reverses T cell exclusion, dampens myeloid cell infiltration and sensitizes PDAC tumors to $\mathrm{T}$ cell-dependent rejection in response to immunotherapy. Interestingly, prostaglandin endoperoxide synthase 2, the gene encoding COX2, is downstream of EPHA2 and its deletion in KPC mice also reverses $\mathrm{T}$ cell exclusion and sensitizes tumors to immunotherapy (52). This genetic phenotype can be largely reproduced with COX2 inhibitors in concert with immunotherapy, revealing a potentially tractable clinically translatable strategy. Another key interaction in the pancreatic cancer TME is the interaction between cancer-associated fibroblasts (CAFs) and stellate cells and myeloid cells. CAFs mold the extracellular matrix (ECM) and provide survival and migration signals to cancer cells, and hinder drug delivery and potentially effector $\mathrm{T}$ cell infiltration $(53,54)$. CAFs themselves secrete cytokines and chemokines and can regulate the immune cell milieu, recruit immune-suppressive cells, and inhibit effector cells $(55,56)$. There is great phenotypic and functional heterogeneity in PDAC CAFs, including one subpopulation with antigen-presentation function (57). These findings provide new therapeutic opportunities, e.g., administration of vitamin D receptor ligand alters inflammation and fibrosis in pancreatitis and tumor stroma (58).

\section{TARGETING MYELOID CHEMOATTRACTANTS FOR IMMUNOTHERAPY}

As illustrated above, tumor-intrinsic chemoattractants recruit myeloid cells into the PDAC TME, block T cell priming, infiltration and effector function, and enable tumor immune escape and growth (Figure 1). Importantly, this myeloid network is operative early in neoplastic development, often before invasion - a pathological stage well-characterized in the spontaneous KPC model. Importantly, the tumor promoting effects of myeloid cells are reversible as robust depletion of myeloid cells accomplished with an engineered toxin prevents both initiation and growth of mutant Kras-driven PDAC in mice (59).

Given T cell exclusion is an early and robust feature of PDAC, at least in the KPC model, the paradigm of immune editing is to be reconsidered because there is no or little Darwinian pressure from a $\mathrm{T}$ cell attack from which tumors must escape (11). Such a paradigm paradoxically implies tumors remain sensitive to $\mathrm{T}$ cells following progression from non-invasive to invasive cancer should the barrier of myeloid immunosuppression be removed (15). The lack of immune editing perhaps explains why PD-L1, upregulated in response to IFN-gamma from T cells, is expressed at low levels in the majority of PDAC tumors (13). In PDAC patients, unleashed $\mathrm{T}$ cell immunity may actually be quite powerful as evidenced by the correlation between a PD-L1 $1^{\text {low }} / \mathrm{CD} 8^{\text {high }}$ tumor sub-phenotype and positive prognosis in PDAC (60) as well as improved overall survival in the few resectable patients who naturally develop strong $\mathrm{T}$ cell immunity to PDAC compared to patients in whom $\mathrm{T}$ responses are minimal to absent (61).

How can the myeloid checkpoint be exploited to unleash antitumor $\mathrm{T}$ cells of clinical significance? One approach is to eliminate TAMs and other myeloid cells directly (50). In KPC mice, eight methodologies - ranging from clodronate to antibodies to combinations - fail to deeply or durably deplete TAMs (even when systemic myeloid cells are successfully depleted in some cases) (8). These disappointing results in mice include the use of antibodies to CSF-1 or CSF-1R. Initial efforts in patients with similar approaches are underway.

A second approach would be to use antibodies that neutralize the dominant cytokine driving myeloid cell accumulation in the TME or the receptor to which it binds. As noted above, this may vary model-to-model, or in the clinic, patient-by-patient requiring precision immune profiling to select the optimal neutralizing antibody or antibodies. Anti-cytokine antibodies 
are increasingly among FDA approved drugs for inflammatory conditions. Among clinical-grade but still experimental antibodies, anti-GM-CSF or anti-CSF1-R antibodies would be logical and reasonable in PDAC patients whose tumors express these cytokines. However, because some cytokines also play other roles in promoting immunity including against pathogens (e.g., GM-CSF and CXCL1), cytokine neutralization represents a potential double-edged sword and must be carefully considered.

A third strategy would be to inhibit the cancer-promoting pathway or oncogene that is driving the cytokine or chemokine production, representing a more proximal and potentially more tumor-specific approach. One example would be to inhibit mutant Kras. Mutant Kras has long been an elusive oncologic target, but recently novel Kras inhibitors are providing new hope toward this possibility (62), although the applicability of KrasG12C inhibitors to PDAC patients is largely limited by the rare prevalence of this mutation in this patient population. Nevertheless, inhibition of mutant Kras or its downstream signaling pathways such PI3K may block cytokine production thereby decreasing myeloid cell accumulation in the TME enabling $\mathrm{T}$ cell infiltration and responsiveness to immunotherapy. It is also possible that such inhibition might, as an added benefit, also block PD-L1 expression on PDAC cells themselves (63).

There are of course other features of the crosstalk between tumor cells, myeloid cells, and other elements of the stroma that may be therapeutically targetable, as recently reviewed $(28,64$, $65)$. These approaches have been discussed elsewhere and include blockade of CD47 (66) or FAK1 (67) on tumor cells, as well as PD-L1 (7) or TREM2 on TAMs (68). Of interest, TREM2 on TAMs is upregulated by GM-CSF and CSF- 1 in certain models (69).

Finally, tumor-derived cytokines represent just one of a growing number of examples of tumor-intrinsic mechanisms of immune suppression. Examples are well-described elsewhere (70-72) and represent immune checkpoints beyond CTLA-4 and PD-1 just like the chemoattractants described here.

\section{REFERENCES}

1. Coussens LM, Werb Z. Inflammation and cancer. Nature (2002) 420:860-7. doi: $10.1038 /$ nature 01322

2. Bottazzi B, Polentarutti N, Acero R, Balsari A, Boraschi D, Ghezzi P, et al. Regulation of the macrophage content of neoplasms bychemoattractants. Science (1983) 220:210-2. doi: 10.1126/science.6828888

3. Bear AS, Vonderheide RH, O'Hara MH. Challenges and opportunities for pancreatic cancer immunotherapy. Cancer Cell (2020). doi: 10.1016/ j.ccell.2020.08.004

4. O’Reilly EM, Oh DY, Dhani N, Renouf DJ, Lee MA, Sun W, et al. Durvalumab with or without tremelimumab for patients with metastatic pancreatic ductal adenocarcinoma: A phase 2 randomized clinical trial. JAMA Oncol (2019) 5:1431-8. doi: 10.1001/jamaoncol.2019.1588

5. Le DT, Durham JN, Smith KN, Wang H, Bartlett BR, Aulakh LK, et al. Mismatch repair deficiency predicts response of solid tumors to PD-1 blockade. Science (2017) 357:409-13. doi: 10.1126/science.aan6733

6. Rahib L, Smith BD, Aizenberg R, Rosenzweig AB, Fleshman JM, Matrisian LM. Projecting cancer incidence and deaths to 2030: The unexpected burden of thyroid,liver, and pancreas cancers in the united states. Can Res (2014) 74:2913-21. doi: 10.1158/0008-5472.CAN-14-0155

7. Winograd R, Byrne KT, Evans RA, Odorizzi PM, Meyer AR, Bajor DL, et al. Induction of t-cell immunity overcomes complete resistance to PD-1 and

\section{CONCLUSIONS}

Preclinical studies using mouse models of PDAC uncover a rich network of tumor-derived cytokines and chemokines that drive the recruitment of myeloid cells to the TME, including TAMs and MDSCs. These cells block the influx and priming of T cells, contributing to $\mathrm{T}$ cell exclusion. Genetic or pharmacologic disruption of this chemokine/cytokine network converts the TME to T cell-high and sensitizes tumors to immunotherapy. Thus, neutralization of such tumor-derived factors or their receptors - or potentially inhibiting the tumor-intrinsic pathways that drive their production - represents a strategy to address the "myeloid immune checkpoint" not only in PDAC but also potentially other tumor types. Efforts to test this hypothesis in patients remain nascent.

\section{AUTHOR CONTRIBUTIONS}

$\mathrm{RV}$ conceived the project, and both authors wrote and edited the manuscript. All authors contributed to the article and approved the submitted version.

\section{FUNDING}

Support from NCI grants R01 CA217176, P01 CA210944, K12 CA076931, P30 CA016520, and the Parker Institute for Cancer Immunotherapy.

\section{ACKNOWLEDGMENTS}

The author acknowledges valuable discussions with Drs. Katelyn Byrne, Jinyang Li, Nune Markosyan, and Ben Stanger.

CTLA-4 blockade and improves survival in pancreatic carcinoma. Cancer Immunol Res (2015) 3:399-411. doi: 10.1158/2326-6066.CIR-14-0215

8. Byrne KT, Vonderheide RH. CD40 stimulation obviates innate sensors and drives t cell immunity in cancer. Cell Rep (2016) 15:2719-32. doi: 10.1016/ j.celrep.2016.05.058

9. Clark CE, Hingorani SR, Mick R, Combs C, Tuveson DA, Vonderheide RH. Dynamics of the immune reaction to pancreatic cancer from inception to invasion. Cancer Res (2007) 67:9518-27. doi: 10.1158/0008-5472.CAN-070175

10. Clark CE, Beatty GL, Vonderheide RH. Immunosurveillance of pancreatic adenocarcinoma: Insights from genetically engineered mouse models of cancer. Cancer Lett (2009) 279:1-7. doi: 10.1016/j.canlet. 2008.09.037

11. Evans RA, Diamond MS, Rech AJ, Chao T, Richardson MW, Lin JH, et al. Lack of immunoediting in murine pancreatic cancer reversed with neoantigen. JCI Insight (2016) 1:e88328. doi: 10.1172/jci.insight.88328

12. Soares KC, Rucki AA, Wu AA, Olino K, Xiao Q, Chai Y, et al. PD-1/PD-L1 blockade together with vaccine therapy facilitates effector T-cell infiltration into pancreatic tumors. J Immunother (2015) 38:1-11. doi: 10.1097/ CJI.0000000000000062

13. Balli D, Rech AJ, Stanger BZ, Vonderheide RH. Immune cytolytic activity stratifies molecular subsets of human pancreatic cancer. Clin Cancer Res (2017) 23:3129-38. doi: 10.1158/1078-0432.CCR-16-2128 
14. Tsujikawa T, Crocenzi T, Durham JN, Sugar EA, Wu AA, Onners B, et al. Evaluation of cyclophosphamide/gvax pancreas followed by listeriamesothelin (CRS-207) with or without nivolumab in patients with pancreatic cancer. Clin Cancer Res (2020) 26:3578-88. doi: 10.1158/10780432.CCR-19-3978

15. Vonderheide RH. The immune revolution: A case for priming, not checkpoint. Cancer Cell (2018) 33:563-9. doi: 10.1016/j.ccell.2018.03.008

16. Rech AJ, Dada H, Kotzin JJ, Henao-Mejia J, Minn AJ, Twyman-Saint Victor C, et al. Radiotherapy and CD40 activation separately augment immunity to checkpoint blockade in cancer. Cancer Res (2018) 78:4282-91. doi: 10.1158/ 0008-5472.CAN-17-3821

17. Yasmin-Karim S, Bruck PT, Moreau M, Kunjachan S, Chen GZ, Kumar R, et al. Radiation and local anti-CD40 generate an effective in situ vaccine in preclinical models of pancreatic cancer. Front Immunol (2018) 9:2030. doi: 10.3389/fimmu.2018.02030

18. Vonderheide RH. CD40 agonist antibodies in cancer immunotherapy. Annu Rev Med (2020) 71:47-58. doi: 10.1146/annurev-med-062518-045435

19. Blando J, Sharma A, Higa MG, Zhao H, Vence L, Yadav SS, et al. Comparison of immune infiltrates in melanoma and pancreatic cancer highlights VISTA as a potential target in pancreatic cancer. Proc Natl Acad Sci U S A (2019) 116:1692-7. doi: 10.1073/pnas.1811067116

20. Gao J, Ward JF, Pettaway CA, Shi LZ, Subudhi SK, Vence LM, et al. VISTA is an inhibitory immune checkpoint that is increased after ipilimumab therapy in patients with prostate cancer. Nat Med (2017) 23:551-5. doi: 10.1038/ nm. 4308

21. Long GV, Dummer R, Hamid O, Gajewski TF, Caglevic C, Dalle S, et al. Epacadostat plus pembrolizumab versus placebo plus pembrolizumab in patients with unresectable or metastatic melanoma (ECHO-301/ KEYNOTE-252): A phase 3, randomised, double-blind study. Lancet Oncol (2019) 20:1083-97. doi: 10.1016/S1470-2045(19)30274-8

22. Bayne LJ, Beatty GL, Jhala N, Clark CE, Rhim AD, Stanger BZ, et al. Tumorderived granulocyte-macrophage colony-stimulating factor regulates myeloid inflammation and T cell immunity in pancreatic cancer. Cancer Cell (2012) 21:822-35. doi: 10.1016/j.ccr.2012.04.025

23. Pylayeva-Gupta Y, Lee KE, Hajdu CH, Miller G, Bar-Sagi D. Oncogenic KRAS-induced GM-CSF production promotes the development of pancreatic neoplasia. Cancer Cell (2012) 21:836-47. doi: 10.1016/j.ccr.2012.04.024

24. Beatty GL, Chiorean EG, Fishman MP, Saboury B, Teitelbaum UR, Sun W, et al. CD40 agonists alter tumor stroma and show efficacy against pancreatic carcinoma in mice and humans. Science (2011) 331:1612-6. doi: 10.1126/ science. 1198443

25. Panni RZ, Herndon JM, Zuo C, Hegde S, Hogg GD, Knolhoff BL, et al. Agonism of CD11b reprograms innate immunity to sensitize pancreatic cancer to immunotherapies. Sci Transl Med (2019) 11(499):eaau9240. doi: 10.1126/scitranslmed.aau9240

26. Lavin Y, Kobayashi S, Leader A, Amir ED, Elefant N, Bigenwald C, et al. Innate immune landscape in early lung adenocarcinoma by paired single-cell analyses. Cell (2017) 169:750-765 e17. doi: 10.1016/j.cell.2017.04.014

27. Gubin MM, Esaulova E, Ward JP, Malkova ON, Runci D, Wong P, et al. Highdimensional analysis delineates myeloid and lymphoid compartment remodeling during successful immune-checkpoint cancer therapy. Cell (2018) 175:1443. doi: 10.1016/j.cell.2018.11.003

28. Jahchan NS, Mujal AM, Pollack JL, Binnewies M, Sriram V, Reyno L, et al. Tuning the tumor myeloid microenvironment to fight cancer. Front Immunol (2019) 10:1611. doi: 10.3389/fimmu.2019.01611

29. Bronte V, Brandau S, Chen SH, Colombo MP, Frey AB, Greten TF, et al. Recommendations for myeloid-derived suppressor cell nomenclature and characterization standards. Nat Commun (2016) 7:12150. doi: 10.1038/ ncomms 12150

30. Gabrilovich DI, Ostrand-Rosenberg S, Bronte V. Coordinated regulation of myeloid cells by tumours. Nat Rev Immunol (2012) 12:253-68. doi: 10.1038/ nri3175

31. Coussens LM, Zitvogel L, Palucka AK. Neutralizing tumor-promoting chronic inflammation: A magic bullet? Science (2013) 339:286-91. doi: 10.1126/ science. 1232227

32. Li J, Byrne KT, Yan F, Yamazoe T, Chen Z, Baslan T, et al. Tumor cellintrinsic factors underlie heterogeneity of immune cell infiltration and response to immunotherapy. Immunity (2018) 49:178-193 e7. doi: 10.1016/ j.immuni.2018.06.006

33. Lin JH, Huffman AP, Wattenberg MM, Walter DM, Carpenter EL, Feldser DM, et al. Type 1 conventional dendritic cells are systemically dysregulated early in pancreatic carcinogenesis. J Exp Med (2020) 217(8):e20190673. doi: $10.1084 /$ jem. 20190673

34. Chao T, Furth EE, Vonderheide RH. CXCR2-dependent accumulation of tumor-associated neutrophils regulates T-cell immunity in pancreatic ductal adenocarcinoma. Cancer Immunol Res (2016) 4:968-82. doi: 10.1158/23266066.CIR-16-0188

35. Steele CW, Karim SA, Leach JDG, Bailey P, Upstill-Goddard R, Rishi L, et al. CXCR2 inhibition profoundly suppresses metastases and augments immunotherapy in pancreatic ductal adenocarcinoma. Cancer Cell (2016) 29:832-45. doi: 10.1016/j.ccell.2016.04.014

36. Seifert L, Werba G, Tiwari S, Giao Ly NN, Alothman S, Alqunaibit D, et al. The necrosome promotes pancreatic oncogenesis via CXCL1 and mincleinduced immune suppression. Nature (2016) 532:245-9. doi: 10.1038/ nature 17403

37. Sanford DE, Belt BA, Panni RZ, Mayer A, Deshpande AD, Carpenter D, et al. Inflammatory monocyte mobilization decreases patient survival in pancreatic cancer: A role for targeting the CCL2/CCR2 axis. Clin Cancer Res (2013) 19:3404-15. doi: 10.1158/1078-0432.CCR-13-0525

38. Zhu Y, Knolhoff BL, Meyer MA, Nywening TM, West BL, Luo J, et al. CSF1/ CSF1R blockade reprograms tumor-infiltrating macrophages and improves response to T-cell checkpoint immunotherapy in pancreatic cancer models. Cancer Res (2014) 74:5057-69. doi: 10.1158/0008-5472.CAN-13-3723

39. Candido JB, Morton JP, Bailey P, Campbell AD, Karim SA, Jamieson T, et al. CSF1R $(+)$ macrophages sustain pancreatic tumor growth through $\mathrm{T}$ cell suppression and maintenance of key gene programs that define the squamous subtype. Cell Rep (2018) 23:1448-60. doi: 10.1016/ j.celrep.2018.03.131

40. Saung MT, Muth S, Ding D, Thomas DL,2, Blair AB, Tsujikawa T, et al. Targeting myeloid-inflamed tumor with anti-CSF1R antibody expands CD137+ effector T-cells in the murine model of pancreatic cancer. J Immunother Cancer (2018) 6:118. doi: 10.1186/s40425-018-0435-6

41. Vonderheide RH. Tumor-promoting inflammatory networks in pancreatic neoplasia: Another reason to loathe KRAS. Cancer Cell (2014) 25:553-4. doi: 10.1016/j.ccr.2014.04.020

42. Aiello NM, Bajor DL, Norgard RJ, Sahmoud A, Bhagwat N, Pham MN, et al. Metastatic progression is associated with dynamic changes in the local microenvironment. Nat Commun (2016) 7:12819. doi: 10.1038/ncomms12819

43. Le DT, Wang-Gillam A, Picozzi V, Greten TF, Crocenzi T, Springett G, et al. Safety and survival with GVAX pancreas prime and listeria monocytogenesexpressing mesothelin (CRS-207) boost vaccines for metastatic pancreatic cancer. J Clin Oncol (2015) 33:1325-33. doi: 10.1200/JCO.2014.57.4244

44. Lesina M, Wormann SM, Morton J, Diakopoulos KN, Korneeva O, Wimmer M, et al. RelA regulates CXCL1/CXCR2-dependent oncogene-induced senescence in murine Kras-driven pancreatic carcinogenesis. J Clin Invest (2016) 126:291932. doi: $10.1172 /$ JCI86477

45. Ijichi H, Chytil A, Gorska AE, Aakre ME, Bierie B, Tada M, et al. Inhibiting CXCR2 disrupts tumor-stromal interactions and improves survival in a mouse model of pancreatic ductal adenocarcinoma. J Clin Invest (2011) 121:4106-17. doi: 10.1172/JCI42754

46. Graves DT, Jiang YL, Williamson MJ, Valente AJ. Identification of monocyte chemotactic activity produced by malignant cells. Science (1989) 245:1490-3. doi: $10.1126 /$ science. 2781291

47. Negus RP, Stamp GW, Relf MG, Burke F, Malik ST, Bernasconi S, et al. The detection and localization of monocyte chemoattractant protein-1 (MCP-1) in human ovarian cancer. J Clin Invest (1995) 95:2391-6. doi: 10.1172/JCI117933

48. Pausch TM, Aue E, Wirsik NM, Freire Valls A, Shen Y, Radhakrishnan P, et al. Metastasis-associated fibroblasts promote angiogenesis in metastasized pancreatic cancer via the CXCL8 and the CCL2 axes. Sci Rep (2020) 10:5420. doi: 10.1038/s41598-020-62416-x

49. Noel M, O’Reilly EM, Wolpin BM, Ryan DP, Bullock AJ, Britten CD, et al. Phase $1 \mathrm{~b}$ study of a small molecule antagonist of human chemokine (c-c motif) receptor 2 (PF-04136309) in combination with nab-paclitaxel/ gemcitabine in first-line treatment of metastatic pancreatic ductal 
adenocarcinoma. Invest New Drugs (2020) 38:800-11. doi: 10.1007/s10637019-00830-3

50. Nywening TM, Belt BA, Cullinan DR, Panni RZ, Han BJ, Sanford DE, et al. Targeting both tumour-associated CXCR2(+) neutrophils and CCR2(+) macrophages disrupts myeloid recruitment and improves chemotherapeutic responses in pancreatic ductal adenocarcinoma. Gut (2018) 67:1112-23. doi: 10.1136/gutjnl-2017-313738

51. Hou P, Kapoor A, Zhang Q, Li J, Wu CJ, Li J, et al. Tumor microenvironment remodeling enables bypass of oncogenic kras dependency in pancreatic cancer. Cancer Discovery (2020) 10:1058-77. doi: 10.1158/2159-8290.CD-19-0597

52. Markosyan N, Li J, Sun YH, Richman LP, Lin JH, Yan F, et al. Tumor cellintrinsic EPHA2 suppresses anti-tumor immunity by regulating PTGS2 (COX-2). J Clin Invest (2019) 129:3594-609. doi: 10.1172/JCI127755

53. Olive KP, Jacobetz MA, Davidson CJ, Gopinathan A, McIntyre D, Honess D, et al. Inhibition of hedgehog signaling enhances delivery of chemotherapy in a mouse model of pancreatic cancer. Science (2009) 324:1457-61. doi: 10.1126/ science. 1171362

54. Sahai E, Astsaturov I, Cukierman E, DeNardo DG, Egeblad M, Evans RM, et al. A framework for advancing our understanding of cancer-associated fibroblasts. Nat Rev Cancer (2020) 20:174-86. doi: 10.1038/s41568-019-0238-1

55. Ohlund D, Handly-Santana A, Biffi G, Elyada E, Almeida AS, Ponz-Sarvise M, et al. Distinct populations of inflammatory fibroblasts and myofibroblasts in pancreatic cancer. J Exp Med (2017) 214:579-96. doi: 10.1084/jem.20162024

56. Feig C, Jones JO, Kraman M, Wells RJ, Deonarine A, Chan DS, et al. Targeting CXCL12 from FAP-expressing carcinoma-associated fibroblasts synergizes with anti-PD-L1 immunotherapy in pancreatic cancer. Proc Natl Acad Sci U S A (2013) 110:20212-7. doi: 10.1073/pnas.1320318110

57. Elyada E, Bolisetty M, Laise P, Flynn WF, Courtois ET, Burkhart RA, et al. Cross-species single-cell analysis of pancreatic ductal adenocarcinoma reveals antigen-presenting cancer-associated fibroblasts. Cancer Discov (2019) 9:1102-23. doi: 10.1158/2159-8290.CD-19-0094

58. Sherman MH, Yu RT, Engle DD, Ding N, Atkins AR, Tiriac H, et al. Vitamin D receptor-mediated stromal reprogramming suppresses pancreatitis andenhances pancreatic cancer therapy. Cell (2014) 159:80-93. doi: 10.1016/j.cell.2014.08.007

59. Zhang Y, Velez-Delgado A, Mathew E, Li D, Mendez FM, Flannagan K, et al. Myeloid cells are required for PD-1/PD-L1 checkpoint activation and the establishment of an immunosuppressive environment in pancreatic cancer. Gut (2017) 66:124-36. doi: 10.1136/gutjnl-2016-312078

60. Danilova L, Ho WJ, Zhu Q, Vithayathil T, De Jesus-Acosta A, Azad NS, et al. Programmed cell death ligand-1 (PD-L1) and CD8 expression profiling identify an immunologic subtype of pancreatic ductal adenocarcinomas with favorable survival. Cancer Immunol Res (2019) 7:886-95. doi: 10.1158/2326-6066.CIR-18-0822

61. Balachandran VP, Luksza M, Zhao JN, Makarov V, Moral JA, Remark R, et al. Identification of unique neoantigen qualities in long-term survivors of pancreatic cancer. Nature (2017) 551:512-6. doi: 10.1038/nature24462

62. Canon J, Rex K, Saiki AY, Mohr C, Cooke K, Bagal D, et al. The clinical KRAS (G12C) inhibitor AMG 510 drives anti-tumour immunity. Nature (2019) 575:217-23. doi: 10.1038/s41586-019-1694-1
63. Coelho MA, de Carne Trecesson S, Rana S, Zecchin D, Moore C, MolinaArcas M, et al. Oncogenic RAS signaling promotes tumor immunoresistance by stabilizing PD-L1 mrna. Immunity (2017) 47:1083-1099 e6. doi: 10.1016/ j.immuni.2017.11.016

64. Ho WJ, Jaffee EM, Zheng L. The tumour microenvironment in pancreatic cancer - clinical challenges and opportunities. Nat Rev Clin Oncol (2020) 17: 527-40. doi: 10.1038/s41571-020-0363-5

65. Balachandran VP, Beatty GL, Dougan SK. Broadening the impact of immunotherapy to pancreatic cancer: Challenges and opportunities. Gastroenterology (2019) 156:2056-72. doi: 10.1053/j.gastro.2018.12.038

66. Feng M, Jiang W, Kim BYS, Zhang CC, Fu YX, Weissman IL. Phagocytosis checkpoints as new targets for cancer immunotherapy. Nat Rev Cancer (2019) 19:568-86. doi: 10.1038/s41568-019-0183-z

67. Jiang H, Hegde S, Knolhoff BL, Zhu Y, Herndon JM, Meyer MA, et al. Targeting focal adhesion kinase renders pancreatic cancers responsive to checkpoint immunotherapy. Nat Med (2016) 22:851-60. doi: 10.1038/ nm. 4123

68. Molgora M, Esaulova E, Vermi W, Hou J, Chen Y, Luo J, et al. TREM2 modulation remodels the tumor myeloid landscape enhancing anti-PD-1 immunotherapy. Cell (2020) 182:886-900. doi: 10.1016/j.cell.2020.07.013

69. Cella M, Buonsanti C, Strader C, Kondo T, Salmaggi A, Colonna M. Impaired differentiation of osteoclasts in TREM-2-deficient individuals. J Exp Med (2003) 198:645-51. doi: 10.1084/jem.20022220

70. Marin-Acevedo JA, Dholaria B, Soyano AE, Knutson KL, Chumsri S, Lou Y. Next generation of immune checkpoint therapy in cancer: New developments and challenges. J Hematol Oncol (2018) 11:39. doi: 10.1186/s13045-018-0582-8

71. Kalbasi A, Ribas A. Tumour-intrinsic resistance to immune checkpoint blockade. Nat Rev Immunol (2020) 20:25-39. doi: 10.1038/s41577-0190218-4

72. Saleh R, Elkord E. Acquired resistance to cancer immunotherapy: Role of tumor-mediatedimmunosuppression. Semin Cancer Biol (2020) 65:13-27.doi: 10.1016/j.semcancer.2019.07.017

Conflict of Interest: RV reports having received consulting fees or honoraria from Celldex, Lilly, Medimmune, and Verastem; and research funding from Apexigen, Fibrogen, Inovio, Janssen, and Lilly. He is an inventor on licensed patents relating to cancer cellular immunotherapy and receives royalties from Children's Hospital Boston for a licensed research-only monoclonal antibody.

The remaining author declares that the research was conducted in the absence of any commercial or financial relationships that could be construed as a potential conflict of interest.

Copyright (๑) 2020 Vonderheide and Bear. This is an open-access article distributed under the terms of the Creative Commons Attribution License (CC BY). The use, distribution or reproduction in other forums is permitted, provided the original author(s) and the copyright owner(s) are credited and that the original publication in this journal is cited, in accordance with accepted academic practice. No use, distribution or reproduction is permitted which does not comply with these terms. 